\title{
X-ray Reverberation Observational Modelling in Active Galactic Nuclei
}

\author{
M. D. Caballero-Garcia*1,M. Dovčiak ${ }^{1}$, M. Bursa ${ }^{1}$, I. Papadakis ${ }^{2,3}$, V. Karas ${ }^{1}$ \\ ${ }^{1}$ Astronomical Institute of the Academy of Sciences, Boční II 1401, CZ-14100 Praha 4, \\ Czech Republic, E-mail: garcia@asu.cas.cz \\ ${ }^{2}$ Department of Physics and Institute of Theoretical and Computational Physics, \\ University of Crete, GR-71003 Heraklion, Greece \\ ${ }^{3}$ Foundation for Research and Technology - Hellas, IESL, Voutes, GR-7110 Heraklion, \\ Greece
}

X-ray reverberation in Active Galactic Nuclei, believed to be the result of the reprocessing of coronal photons by the underlying accretion disc, has allowed us to probe the properties of the inner-most regions of the accretion flow and the central black hole. Our current model (KYNREFREV) computes the time-dependent reflection spectra of the disc as a response to a flash of primary power-law radiation from a point source corona located on the axis of the black hole accretion disc (lamp-post geometry). Full relativistic effects are taken into account. The ionization of the disc is set for each radius according to the amount of the incident primary flux and the density of the accretion disc. We detect wavy residuals around the best-fit reverberation model time lags at high frequencies. This result suggests that the simple lamp-post geometry does not fully explain the X-ray source/disc configuration in Active Galactic Nuclei. There has been a noticeable progress into the development of codes for extended coronae (Wilkins et al. 2016, Chainakun \& Young 2017, Taylor \& Reynolds, 2018a,b). Indeed, the model from Chainakun \& Young (2017), consisting of two axial point sources illuminating an accretion disc that produce the reverberation lags is able to reproduce the observed time-lag versus frequency spectra. The goal of this paper is to observationally justify the need for an extended corona in order to provide (in the near future) with a mathematical formulation of a model for an extended corona in its simplest form.

Accretion Processes in Cosmic Sources - II - APCS2018

3-8 September 2018

Saint Petersburg, Russian Federation

${ }^{*}$ Speaker. 


\section{Introduction}

The first theoretical predictions and simulations of the time evolving echoes of fluorescent lines due to reflection from relativistic accretion discs around Schwarzschild black holes (BHs) come from the 90s [1,2]. Since then the models were improved to include rotating Kerr BHs as well as more realistic reflection spectra where ionisation of the disc is considered. Some of these models compute not only the light curves and dynamical (time evolving) spectra but also the resulting frequency dependent lags between different energy bands and energy dependent lags at a given frequency and thus produce results directly comparable with the observational data. Currently the most advanced modelling is performed by several groups around the world (e.g. $[3,4,5,6])$. Each of these models is suited for different use. The code by Wilkins et al. [4] is suitable for investigations of photon delays considering extended corona, where the fluctuations inside the corona are studied. We notice that in these models for X-ray reverberation there is not any physical heating/cooling mechanism assumed for the corona (for simplicity). The model from Chainakun \& Young [5] may be used inside the spectral fitting package XSPEC [7] with the possibility to define an extended corona as well, however, large data tables have to be pre-calculated first to be able to fit for all the parameters these types of models have and these computations are quite heavy on computational time (huge servers with many processing cores are needed). On the other side the model developed in our group (Dovčiak et al. [6]) aims to be suited for fast fitting inside XSPEC (every run takes a few seconds only in a single portable computer) on the expense of a simplified lamp-post geometry provided. This XSPEC model is publicly available ${ }^{1}$.

Models of X-ray reverberation from extended coronae are developed from general relativistic ray tracing simulations. Reverberation lags between correlated variability in the directly observed continuum emission and that reflected from the accretion disc arise due to the additional light travel time between the corona and reflecting disc. X-ray reverberation is detected from an increasing sample of Seyfert galaxies and a number of common properties are observed, including a transition from the characteristic reverberation signature at high frequencies to a hard lag within the continuum component at low frequencies (see e.g. [8] and references therein).

This paper is the continuation of a previous work [9], which focused on preliminary results obtained by using the code KYNREFREV by Dovčiak et al. [6]. This model takes into account a treatment of all the general relativistic effects for accretion discs and light bending effects in the disc-corona geometry considering the lamp-post geometry. Whilst in previous works $[8,9]$ we focused on the determination of the spin of the $\mathrm{BH}$, here we compare our results in the context of discussion of possible extended coronal geometries. Sec. 1.1 shows a brief description of the model, Sec. 2 provides the description of models and results obtained by the use of them considering an extended corona geometry, in Sec. 3 we briefly discuss and compare our results with them. Finally, in Sec. 4 we summarize the obtained results and present our plans for future developments in order to provide for a mathematical formulation of a model for an extended corona in its simplest form.

\subsection{The lamp-post model (Dovčiak et al. [6] KYNREFREV)}

Our code (KYNREFREV; [6]) takes into account a treatment of all the general relativistic ef-

\footnotetext{
${ }^{1}$ https://projects.asu.cas.cz/stronggravity/kynreverb
} 
fects for accretion discs and light bending effects in the disc-corona geometry. Time-lags versus frequency and energy can be produced and fitted, either in XSPEC or using another suitable tool. This model computes the X-ray emission from an accretion disc in the lamp-post geometry where the point-like patch of corona (of zero extension) located on the axis above the black hole illuminates a geometrically thin Keplerian accretion disc in the equatorial plane. The main physical parameters of the model are the $\mathrm{BH}$ mass and angular momentum, the height of the X-ray source and the observer viewing angle. The re-processing in the disc is modelled by using the REFLIONX tables [10]. The radial ionisation profile is computed from the illuminating flux and the disc ionization is parametrised as a radial power-law. The reflecting disc region (inner and outer edge and azimuthal segment) as well as the circular obscuring cloud can be set in the model. The power-law hard lag for frequency dependence is also included directly in the model.

\section{Existing models for an extended corona}

\subsection{The horizontally and vertically extended corona (Wilkins et al. [4]); model A)}

This model has been motivated in order to explain the low-frequency hard lags observed in the time-lag spectra together with the reverberation lags at high frequencies with the same physical model. So far, there was only an emipirical prescription for the description of the hard-lags. Furthermore, this model has the aim to explain the presence of an ubiquitous minimum in the reverberation time-lag versus energy spectra at $3 \mathrm{keV}$ observed in Seyfert galaxies.

Wilkins et al. [4] model the reverberation time-lags as produced from extended coronae both over the surface of the disc and vertically. In the model from Wilkins et al. [4] they refer to horizontal and vertical propagations and different physical scenarios are proposed. The corona extends radially at low height over the surface of the disc but with a bright central region in which fluctuations propagate up the black hole rotation axis (see Fig. 1). We refer the reader to Wilkins et al. [4] for a proper description of all the different propagations modes considered by them.

\subsubsection{Comparison with our lamp-post model (KYNREFREV)}

We have produced time-lags versus frequency and energy (the latter at the frequency of $10^{-4} \mathrm{~Hz}$ ) and the average spectrum (divided by the duration of the flare) for a height of the primary of $5 \mathrm{R}_{\mathrm{g}}$ and using different values for the power index of the radial ionization profile of the disc (parameter q_n $=0,-1,-2)^{2}$. The value for the normalization of the density (parameter $\mathrm{nH} 0$ ) has been set to a canonical value of $10^{15} \mathrm{~cm}^{-3} 3$. The results are shown in Fig. 2. From the plots we can see that when the radial "density" distribution is steeper, the inner disc reflection component dominates. This translates into an increase of the effects due to our relativistic model occurring in the innermost regions around the $\mathrm{BH}$. Due to the fact that the radial decrease of ionisation is smaller for

\footnotetext{
${ }^{2}$ The one power-law index distribution of density is not realistic. We are working on more realistic assumptions for the density distribution (work in progress).

${ }^{3}$ Since REFLIONX tables are computed only for one value of density, we really cannot change the density, i.e. by changing the density we mean that we effectively change the ionisation of the disc (from the fact that $\xi(r, \phi) \propto \mathrm{F}(\mathrm{r}, \phi) / \mathrm{n}(\mathrm{r}, \phi)$ (i.e. note that the dependence of ionization on $\phi$ are due to the GR effects and may sometimes be omitted hereafter), where $\xi$ and $n(r)$ are the ionization and the density profiles, respectively), i.e. the ionisation is not only dependent on the radial profile of illumination (given by the lamp-post geometry) but also by radial profile of density.
} 


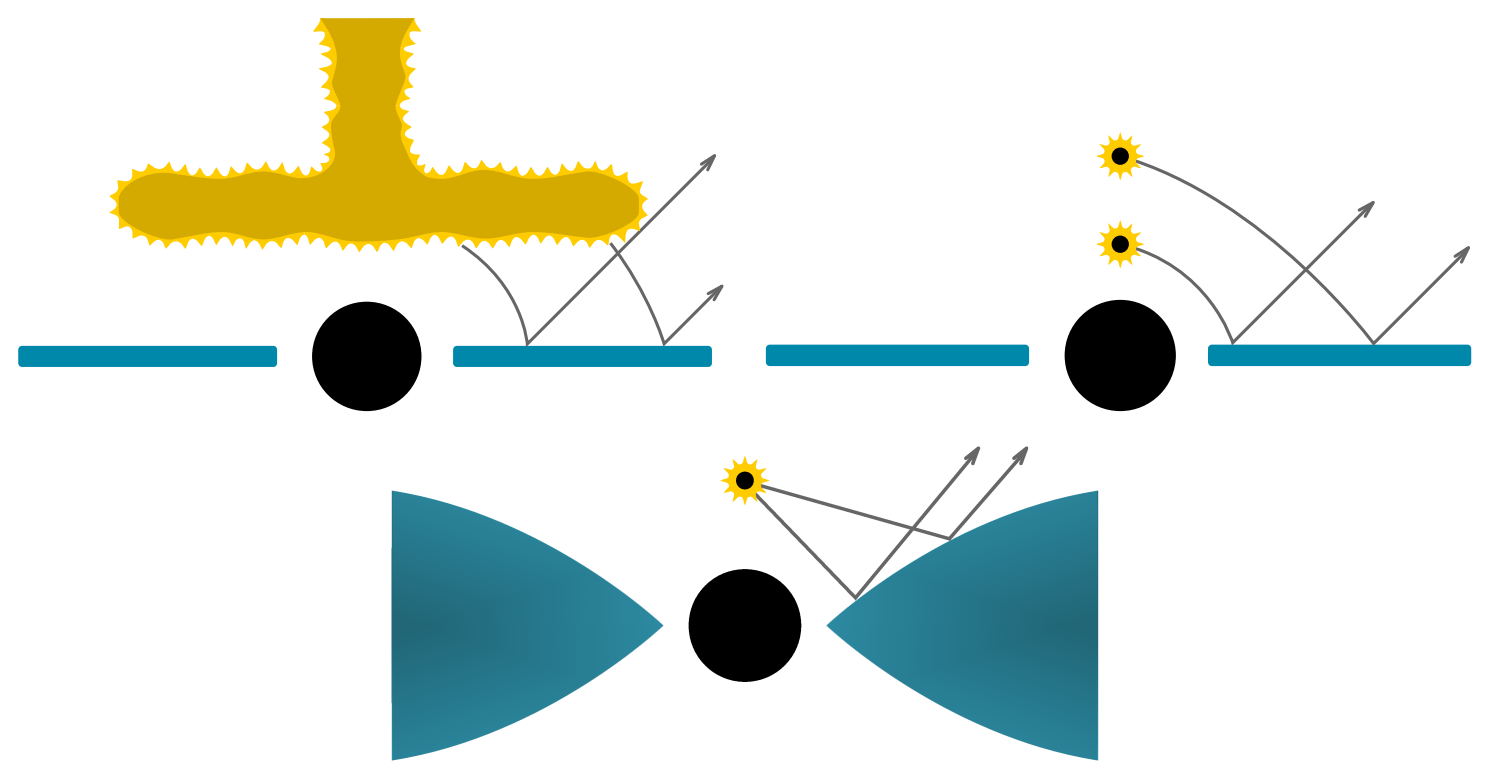

Figure 1: The sketch of the extended corona model used by Wilkins et al. ([4], model A), the two lamp-post coronae used by Chainakun \& Young ([5], model B) and the corona with a geometrically thick accretion disc by Taylor \& Reynolds ([16], model C).

most radii the contribution from inner disc part is more ionised and thus there are more reflected photons especially in the soft band. Therefore, the dilution by primary is smaller and there is an increase in lags (see lag vs. frequency, i.e. top panel, in Fig. 2). These effects are both the increase of the time lags at the highest frequencies $\left(\gtrsim 10^{-4} \mathrm{~Hz}\right)$ and of the red wing of the relativistic $\mathrm{Fe} \mathrm{K}_{\alpha}$ line, which is due to emission from the innermost parts of the accretion disc. This also explains the lag vs. energy dependence, note that more ionised spectra are steeper (due to larger soft excess). Visually, this corresponds to the presence of a pronounced minimum at $\approx 3 \mathrm{keV}$, occurring when the power index is steep (see their Fig. 21, left panel). We will mention about this fact further in Sec. 3 .

We also made a further test of obtaining the absolute time lags (where the whole energy band is used as a reference; by setting $\mathrm{E} 3=-1)$ versus energy at low $\left(\approx 10^{-5} \mathrm{~Hz}\right)$ and high $\left(\approx 10^{-4} \mathrm{~Hz}\right)$ frequencies. We used the same values as in Fig. 2 for the spin, inclination angle, height of the primary source, normalization of the "density" and fixed the radial "density" profile to -2 . The results are shown in Fig. 3. As it can be seen, the lags are larger and have a narrower peak of the relativistic $\mathrm{Fe}_{\alpha}$ line at low frequencies than at high frequencies. This means that at higher frequencies we are tracking the emission from the matter closer to the $\mathrm{BH}$, where the relativistic effects are more important. This broadens the shape of the relativistic $\mathrm{Fe}_{\alpha}$ line, as it can be seen. Further away, the time lags become longer and the relativistic $\mathrm{Fe} \mathrm{K}_{\alpha}$ line becomes peaked, because of the more negligible relativistic effects. This is consistent with the findings of [11] (see their Fig. 7).

\subsection{The two blobs extended corona (Chainakun \& Young [5]); model B)}

Chainakun \& Young ([5]) present an extended coronal model that consists of two axial point 

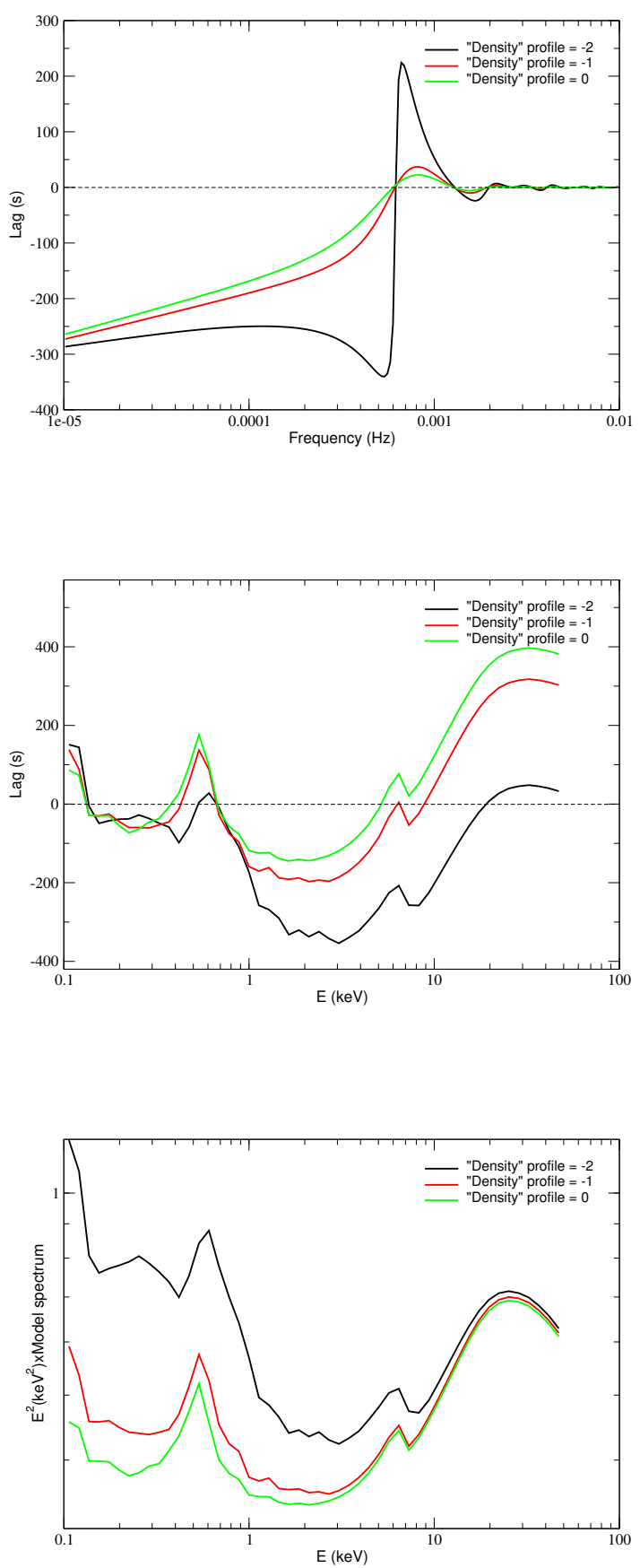

Figure 2: Variations with the radial "density" profile power-law power index (Top) The dependence of the lag (in seconds) with frequency $(\mathrm{Hz})$ of the emission in the (0.3-0.8 keV) energy range with respect to the (1-3 keV) energy band. (Medium) Lag (in seconds) diluted by primary radiation versus energy (keV) with respect to the (0.1-10 keV) energy band at the frequency of $10^{-4} \mathrm{~Hz}$. (Bottom) Average spectrum (i.e. divided by the flare duration) multiplied by energy in the (0.1-50 keV) energy range. Different radial power-law "density" profile power indexes of -2 (black), -1 (red) and 0 (green) have been considered, with a "density" profile normalization of $10^{15} \mathrm{~cm}^{-3}$. The mass of the $\mathrm{BH}$ is $\mathrm{M}=10^{7} \mathrm{M}_{\odot}$ and the adimensional spin, inclination of the observer and height of the primary source are $\mathrm{a}=1, \theta=30^{\circ}$ and $\mathrm{h}=5 \mathrm{R}_{\mathrm{g}}$, respectively. 

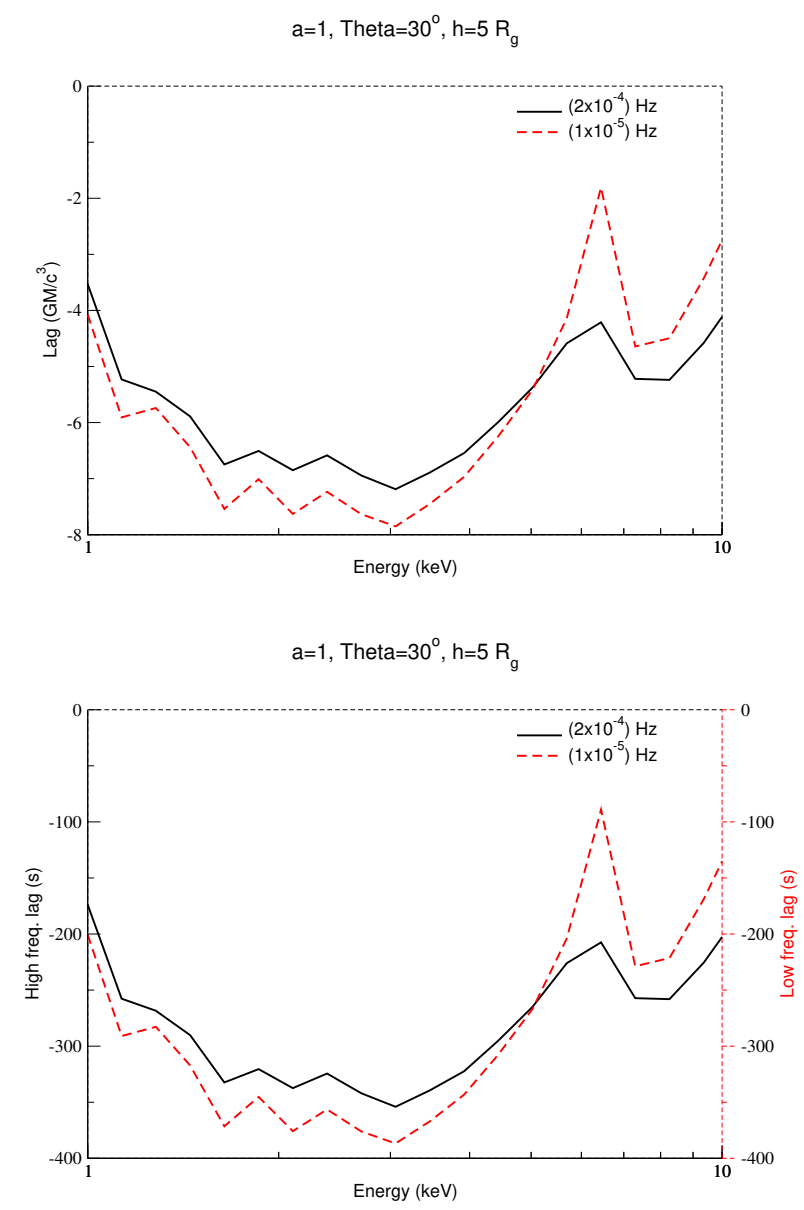

Figure 3: Variations of lag-energy plots with frequency. Lag in gravitational units (top) and in seconds (bottom) diluted by primary radiation versus energy ( $\mathrm{keV}$ ) with respect to the $(0.1-10 \mathrm{keV})$ energy band at high/low frequencies (solid/dashed lines), at the frequencies of $\left(1 \times 10^{-4}\right) \mathrm{Hz}$ and $\left(1 \times 10^{-5}\right) \mathrm{Hz}$, respectively. A radial power-law density profile power index of -2 has been considered, with a normalization of $10^{15} \mathrm{~cm}^{-3}$. The mass of the $\mathrm{BH}$ is $\mathrm{M}=10^{7} \mathrm{M}_{\odot}$ and the adimensional spin, inclination of the observer and height of the primary source are $\mathrm{a}=1, \theta=30^{\circ}$ and $\mathrm{h}=5 \mathrm{R}_{\mathrm{g}}$, respectively.

sources illuminating an accretion disc that produce the reverberation lags (Fig. 1). They assume that these two sources are two lamp-post sources located in the same vertical axis and that both respond independently. Therefore their calculated disc response and the total lags consist of a combination of both source and disc responses. They conclude that their analysis of the energy lag spectra of PG 1244+026 shows that the upper source produces small amounts of reflection and can be interpreted as a relativistic jet.They also suggest that the fluctuations propagating between the two sources of PG 1244+026 are possible but only at near the speed of light.

\subsubsection{Comparison with our lamp-post model (KYNREFREV)}

As we mentioned in Caballero-Garcia et al. [8] the time-lag versus frequency spectra of a sample of Seyfert Active Galactic Nuclei (AGN) show wavy-residuals which are clearly present in the best-fit residuals, in particular in the case of Ark 564 and 1H 0707-495 (see the residual plots in 
Fig. 1 of that paper). These residuals are responsible for the relatively poor statistics of the best-fit models, and suggest that the simple lamp-post geometry (which the KYNREFREV model assumes) may not be an accurate representation of the X-ray/disc configuration in the inner regions of AGNs.

This may not be surprising since the lamp-post geometry, although widely assumed in the literature, is probably a simplification of the real situation. A more complicated model has been recently proposed by Chainakun \& Young [5]. Their model consists of two co-axial point sources illuminating the accretion disc. It assumes that the variations of two X-ray sources are triggered by the same primary variation, but the two sources respond in different ways. The total time-lags are the result of a combination of both the source and disc responses. Their model predictions (see their Fig. 4) appear to be in agreement (qualitatively) with the peaky residuals in our Fig. 1 from [8].

\subsection{The geometrically thick disc and lamp-post and/or flaring corona (Taylor \& Reynolds [16]); model C)}

From the point of view of X-ray spectroscopy Taylor \& Reynolds [15] found that, at the geometrically thin disc limit their model is consistent with other models taking into account the same approximation, but the spectral models start to diverge significantly when $\dot{M}>0.1 \dot{M}_{\text {Edd }}$. With these results, [15] concluded that accretion disc geometry should not be neglected in the detailed modeling of moderate-to-high luminosity AGN reflection spectra, and thus it is reasonable to explore consequences of finite disc thickness in reverberation.

Later Taylor \& Reynolds [16] proposed a new model to explore the effect that accretion disc geometry has on reverberation signatures, assuming a lamp-post configuration but allowing for a finite disc scale height (Fig. 1; i.e. geometrically thick disc).

They also show that a flaring corona (approximated by off-axis point-like flares) would not be sufficient to explain the observed high-frequency lags in AGN if the corona has a very low height. This is consistent with the common view that the corona responsible for the high frequency lag characteristics, such as the $\mathrm{Fe} \mathrm{K \alpha}$ reverberation, must be physically separated from the reprocessing material [17].

\subsubsection{Comparison with our lamp-post model (KYNREFREV)}

As we mentioned in Caballero-Garcia et al. [8] the time-lag versus frequency spectra of a sample of Seyfert Active Galactic Nuclei (AGN) suggest a compact X-ray source, located close to the $\mathrm{BH}$, at a height of approx. 4 gravitational radii and that this result does not depend either on the $\mathrm{BH}$ spin or the disc ionization. Also, the similarity of the wavy-residuals around the best-fit reverberation model time-lags at high frequencies with the model from Chainakun \& Young [5] is in agreement with the fact that the corona has to be physically separated from the accretion disc, in agreement with the model of Taylor \& Reynolds [16].

\section{Discussion}

In this paper we present some interesting scientific outputs obtained by using the KYNREFREV model [6] which deals with X-ray reverberation effects in AGN and can be used in and/or outside XSPEC. This model takes into account all the relativistic effects of the light surrounding the BH 
and a realistic prescription of the accretion disc surrounding it under the lamp-post primary source geometry. We undertake the novel approach of considering a non-constant radial "density", illumination and ionization profiles of the accretion disc, which is likely the most realistic situation. This is done by using a variable ionization parameter $\xi(r, \phi)$ as an input for the REFLIONX tables used in our code. Taking this into account we derive some interesting physical consequences. The first one is that for steep radial "densities" $(\leq-1,-2)$, i.e. for non-constant "density" distributions, the relativistic effects dominate. As a result, the red wing of the relativistic $\mathrm{Fe} \mathrm{K}_{\alpha}$ line and from the so-called "soft-excess" appear broader in the lag-energy spectra when compared to the non-relativistic case (as is the case of the reflection in the outer regions of the disc, where we also expect longer time-delays versus energy). This produces a shift to higher energies of the minimum to $3 \mathrm{keV}$ in the lag-energy spectra, which coincides with the spectral region where the power-law emission from the primary source is dominating, hence the relativistic effects are minimal.

The second one is that the innermost regions are more affected by GR effects than the outer regions. This means that the highest frequency lags (produced closer to the $\mathrm{BH}$ ) have a broader red relativistic $\mathrm{Fe} \mathrm{K}_{\alpha}$ line wing than the low frequency ones (produced further away from the $\mathrm{BH}$ ), which are peaked. This fact agrees with what it was already pointed out in previous studies [11] so we will not discuss it any further.

Both the first and second physical consequences derived by using this model coincide with what it is theoretically expected by taking into account pure GR effects in the most realistic accretion disc scenario (i.e. with variable "density", illumination and ionization radial profile) under the simple lamp-post geometry. More complicated prescriptions of the corona can be considered, as for example the use of extended coronae. This work has been done already [4]. They have developed an X-ray reverberation code taking into account all the effects due to GR under the approximation of a constant "density" profile. They find that, in order to produce the pronounced minimum at $3 \mathrm{keV}$ in the lag-spectra, which has been observed in a few AGN, an horizontally and vertically extended corona is needed. We emphasize here that only a vertically extended corona, with propagations travelling at $v \approx 1 \%$ of the speed of light, under the assumption of constant properties of the disc, can reproduce the observed reverberation lags (see their Fig. 21). We notice that in the case of vertical propagation at speed of light $(v=c)$, the model by Wilkins et al. [4] is not able to reproduce the observed minimum at $3 \mathrm{keV}$ (see their Fig. 21, left panel), that we naturally reproduce with our lamp-post (and ionized disc) model.

\section{Summary and conclusions}

The results of the comparison between our model and these ones can be summarised as follows:

- The time-lags observed from a sample of Seyfert 1 AGN (Caballero-Garcia et al. [8]) show residuals compatible with the two-blobs extended corona model (Chainakun \& Young [5]).

- In the lamp-post approximation and taking into account realistic accretion rates and/or disc thickness of the accretion disc show that very low coronal heights $\left(h \leq 3 r_{\mathrm{g}}\right)$ are not possible(observed) (Taylor \& Reynolds [16]), in agreement also with our findings from a study of the data of a sample of Seyfert 1 AGN (Caballero-Garcia et al. [8]). 
- From all these studies one can say that: there are strong indications from the reverberation $\mathrm{X}$-ray mapping that most of the irradiating photons must come from a source(corona) that is physically separated from the reprocessing material, such as a (zero-extent) lamp-post or an extended jet.

From the total phase of the Fourier transform of the total flux (i.e. both the primary and the reflection continuum are taken into account) one can calculate the time-lag of the signal. This is computed from the total phase at energy bin $\mathrm{E}$ with respect to the total phase at some reference energy bin (see [9]). This can be done from each blob of the corona as emitting in the form of a power-law (for simplicity). We note that any further developments of this formulation will come in future work.

\section{Acknowledgments}

MCG, MD and MB acknowledge support from the Grant Agency of the Czech Republic within the project $n^{\circ} 18-00533 \mathrm{~S}$. MCG acknowledges funding from ESA through a partnership with IAACSIC (Spain).

\section{References}

[1] Stella, L. Natur, 344, 747, (1990)

[2] Campana, S. \& Stella, L. MNRAS, 272, 585, (1995)

[3] Cackett, E. M, et al. MNRAS, 438, 2980, (2014)

[4] Wilkins, D. R., et al. MNRAS, 458, 200, (2016)

[5] Chainakun, P. \& Young, A. J. MNRAS, 465, 3965, (2017)

[6] Dovčiak, M., et al. (in prep.) ApJS, , , (2019)

[7] Arnaud, K. A. Astronomical Society of the Pacific Conference Series, 101, 17, (1996)

[8] Caballero-García, M. D., et al. MNRAS, 480, 2650, (2018)

[9] Caballero-García, M. D.,et al. PoS, APCS, 47, (2016)

[10] Ross, R. R. \& Fabian, A. C., MNRAS, 358, 211, (2005)

[11] Zoghbi, A., et al. MNRAS, 422, 129, (2012)

[12] Martocchia, A. \& Matt, G. MNRAS, 282, 53, (1996)

[13] Reynolds, C. S. \& Begelman, M. C. ApJ, 488, 109, (1997)

[14] Miniutti, G. \& Fabian, A. C. MNRAS, 349, 1435, (2004)

[15] Taylor, C. \& Reynolds, C. S. ApJ, 855, 120, (2018a)

[16] Taylor, C. \& Reynolds, C. S. ApJ, 868, 109, (2018b)

[17] Reis, R. C. \& Miller, J. M. ApJ, 769, 7, (2013) 